\title{
Effects of Dietary and Lighting Conditions on Diurnal Locomotor Activity and Body Temperature in Microminipigs
}

\author{
KAICHIRO TAKEISHI ${ }^{1 *}$, HIROAKI KAWAGUCHI ${ }^{1 *}$, KOHEI AKIOKA $^{2}$, \\ MICHIKO NOGUCHI ${ }^{3}$, EMI ARIMURA ${ }^{1,4}$, MASAHARU ABE $^{1}$, MIHARU USHIKAI $^{1}$, \\ SHINOBU OKITA ${ }^{1}$, AKIHIDE TANIMOTO $^{5}$ and MASAHISA HORIUCHI ${ }^{1}$ \\ Departments of ${ }^{1}$ Hygiene and Health Promotion Medicine, and ${ }^{5}$ Pathology, \\ Graduate School of Medical and Dental Sciences, and ${ }^{2}$ Laboratory of Veterinary Histopathology, \\ Joint Faculty of Veterinary Medicine, Kagoshima University, Kagoshima, Japan; \\ ${ }^{3}$ Laboratory of Theriogenology, Azabu University, Kanagawa, Japan; \\ ${ }^{4}$ Department of Life and Environmental Science, Kagoshima Prefectural College, Kagoshima, Japan
}

\begin{abstract}
The effects of dietary and lighting conditions on diurnal rhythm of locomotor activity (LA) and body temperature (BT) using four adult male microminipigs were investigated. Different feeding times, diet and lighting conditions were applied sequentially for 3 weeks in each phase as follows: Phase I: Morning mealtime, normal diet, 12-h lights on; phase II: mealtime changed to afternoon; phase III: diet changed to high-fat diet; phase IV: lighting changed to 20-h on; and phase V: phase I repeated. LA was measured by an actigraph which was worn on the body of each pig. A BT recording module (Thermochron Type-SL) was implanted in the neck subcutaneously. Phase II increased BT compared with phase I. Phase III increased LA and BT compared with phase II. Phase IV increased LA compared with phase III. LA in phase $V$ was higher compared with phase I. These results can be extrapolated to other diurnal animals such as humans. This study provides an example of the effects of diet and lighting on biological activities in microminipigs under low-invasive procedures measuring LA and BT, leading to low variations in these measures.
\end{abstract}

This article is freely accessible online.

\footnotetext{
*These Authors contributed equally to this study.

Correspondence to: Masahisa Horiuchi, Department of Hygiene and Health Promotion Medicine, Graduate School of Medical and Dental Sciences, Kagoshima University, 8-35-1 Sakuragaoka, Kagoshima 890-8544, Japan. Tel: +81 992755291, Fax: +81 992658434, e-mail: masakun@m.kufm.kagoshima-u.ac.jp
}

Key Words: Diurnal rhythm, lighting conditions, mealtime, swine model, working environments.
In modern society, dietary and lighting conditions such as working environments are highly divergent. This diversity may be linked to morbidity of metabolic diseases, mental health issues, and cancer (1-4). As dietary conditions have changed in modern society, delay in mealtimes and increases in dietary fat content have become more frequent. In terms of lighting conditions, given longer working hours, individuals are exposed to light for longer periods of time. For maintaining health, the effects of dietary and lighting conditions on biological activities should be understood. Many studies on diurnal rhythm in humans have examined changes in locomotor activity (LA) and body temperature (BT) (5-8).

To understand the effects of dietary and lighting conditions on LA and BT, many studies using animal models have also been conducted $(9,10)$. Dietary and lighting conditions may affect the diurnal rhythm in LA and BT through the suprachiasmatic nuclei centrally and internal organs peripherally. The underlying mechanism involves clock genes such as B-cell lymphoma 2 in rodent models ( 9 , 10). However, because rodents are nocturnal animals, caution should be taken when extrapolating the results to humans $(11,12)$. As large mammals, dogs have been used to study the effects of dietary and lighting conditions on LA, showing a diurnal pattern (13); however, these models represent carnivores, although adapted to starch-rich diets, in contrast to the dietary habits of humans $(14,15)$. Pigs as domestic animals have dietary habits (omnivorous) and diurnal behaviors similar to those of humans (16). Therefore, the pig is a suitable animal model for determining the influence of dietary and lighting conditions on LA and BT (17). Here, we examined the effects of dietary and lighting conditions on biological activities using a minipig model, one of the large mammals representing omnivorous and diurnal animals (17). We used microminipigs (Fuji Micra Inc., Shizuoka, Japan), 
a new swine model exhibiting atherosclerosis with a high-fat diet $(18,19)$. The microminipig is registered with the Japanese Ministry of Agriculture, Forestry and Fisheries as a swine that has been recently established as a closed colony. We previously reported the diurnal rhythm in LA in microminipigs (20). Microminipigs are weaned at 3 weeks of age; the reproductive system matures around 6-9 months of age (21). Additionally, developmental BW changes have been reported (22). Based on the maturation of the reproductive system and growth curves, microminipigs at 24 months of age may be equivalent to young adult humans, thus representing this age group.

The significance of the present study is that the procedures evaluating behaviors such as LA and BT are low-invasive, and these pigs at 24 months of age may be equivalent to young adult humans. The longer period of the light-on phase and high-fat diet used are key factors for influencing behavior in young adult humans.

\section{Materials and Methods}

Four male microminipigs were obtained from the breeder and kept individually in a humidity- $(50 \pm 20 \%)$ and temperature-controlled $\left(24 \pm 3^{\circ} \mathrm{C}\right)$ facility with a 12 -h light/dark cycle $(07: 00 \mathrm{~h}$, lights on $)$ as the standard condition. Light intensity during the day was around 300 lux. A standard diet (Kodakara 73; Marubeni Nisshin Feed Inc., Tokyo, Japan) was supplied to the pigs at around 10:00 $\mathrm{h}$ as standard condition. A moderately high fat (MHF) diet was prepared by adding $12 \mathrm{~g}$ of lard and $0.5 \mathrm{~g}$ of cholesterol to $100 \mathrm{~g}$ of the standard diet (19). Energy was calculated using Atwater general factors (23). The energy derived from protein, fat and carbohydrate of the standard diet $(3.20 \mathrm{kcal} / \mathrm{g})$ was $19 \%, 6 \%$ and $75 \%$, respectively. The energy derived from protein, fat and carbohydrate of the MHF diet (3.98 kcal/g) was $13 \%, 34 \%$ and 53\%, respectively. The pigs had free access to water. This study was approved by the Ethics Committee for Animal Experimentation at Kagoshima University (MD 15044 and 15054).

Experimental design. Body weights (BW) were measured each week. During the experimental phases, diet at $3 \%$ of BW was provided. Given that diets were completely consumed each time, the feeding conditions were evaluated as a restricted condition. For the experiments, conditions for each phase were applied sequentially for 3 weeks as follows: Phase I: mealtime at 10:00 h, standard diet, 12-h lights on; phase II: mealtime at 16:00 h, standard diet, 12-h lights on; phase III: mealtime at 16:00 h, MHF diet, 12-h lights on; phase IV: mealtime at 16:00 h, MHF diet, 20-h lights on; and phase V: mealtime at 10:00 h, standard diet, 12-h lights on (same conditions as phase I).

Locomotor activity measured by actigraph. An actigraph (Octagonal Basic Motionlogger; Ambulatory Monitoring, Inc., Ardsley, NY, USA) was worn on the body of each pig for all phases. We used Zero-Crossing Mode for quantification of movement with ACTIONW 2.0 software (Ambulatory Monitoring, Inc., Ardsley, NY, USA) $(8,24)$. Actual activity counts were measured as LA.

Body temperature. Pigs were anesthetized using ketamine (5 $\mathrm{mg} / \mathrm{kg})$, medetomidine $(0.04 \mathrm{mg} / \mathrm{kg})$, and midazolam $(0.2 \mathrm{mg} / \mathrm{kg})$,
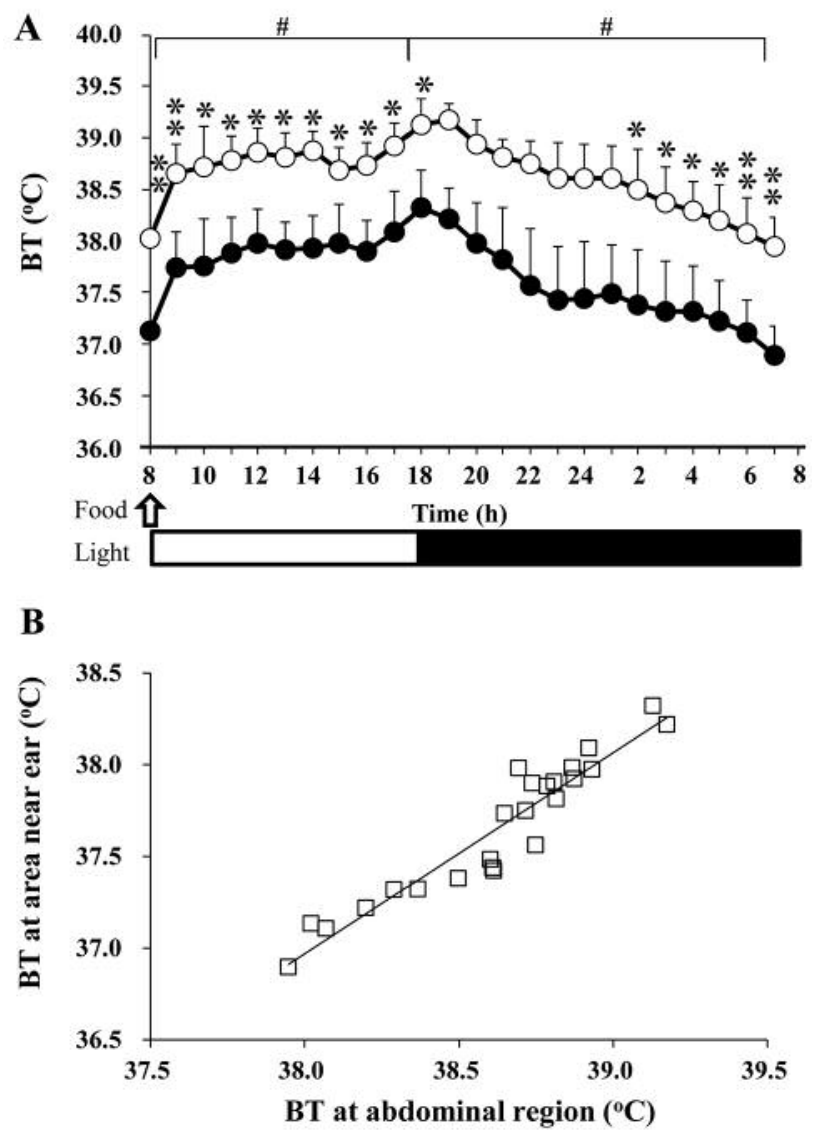

Figure 1. A: Comparison of body temperature (BT) over the course of a day at a position on the abdominal region (open circle) and the neck subcutaneously behind the ear (closed circle). Dietary and lighting conditions are shown in the lower portion of the figure. Arrows represent the mealtime $(08: 00 \mathrm{~h})$. Light and dark conditions (light on at 08:00 $\mathrm{h}$ and light off at 18:00 h) are represented by open and dark bars, respectively. The data represent the mean $+S E$ at each time point of three microminipigs over 1 week. Significantly different at $* p<S E 0.05, * * p<S E 0.01$ between data at the same time point (Student's paired t-test) and at ${ }^{\#} p<0.05$ between respective lighting conditions (two-way ANOVA, repeated measurement, paired). B: The correlation between BT at two locations. Pearson's correlation coefficient $(r)$ was calculated as $0.950(p<0.01)$.

and local anesthesia at the neck (lidocaine). Then the BT recording module (Thermochron Type-SL, KN Laboratories, Inc., Osaka, Japan) was implanted in the neck subcutaneously behind the ear (25). In all studies, BT was recorded once per hour throughout the experiment. Representative data $(\mathrm{N}=3)$ showing the comparison between BT at the neck subcutaneously behind the ear and at the abdominal region $(\mathrm{r}=0.950, p<0.01)$ are presented in Figure 1.

Blood examinations. Blood was collected from the cranial vena cava of each conscious animal at around 09:00 $\mathrm{h}$ before feeding on the second-to-last day of the respective phase to prevent the influence 
Takeishi et al: Dietary and Lighting Conditions Alter Diurnal Rhythm

Table I. Influence of dietary and light conditions on body weight changes, blood glucose, and hormone levels.

\begin{tabular}{lccccc}
\hline Parameter & \multicolumn{4}{c}{ Phase } \\
\cline { 2 - 6 } & I & II & III & IV & V \\
\hline BW change (kg/3 weeks) & $1.3 \pm 0.5$ & $1.3 \pm 0.3$ & $2.0 \pm 0.2$ & $-0.3 \pm 0.1^{* \#}$ & $-0.8 \pm 0.3$ \\
BG (mg/dl) & $110.8 \pm 18.7$ & $133.0 \pm 28.9$ & $158.5 \pm 38.1$ & $106.3 \pm 12.5$ & $98.8 \pm 19.2$ \\
TG (mg/dl) & $20.5 \pm 3.1$ & $23.8 \pm 4.6$ & $33.0 \pm 7.2$ & $11.8 \pm 2.3^{\#}$ & $14.0 \pm 2.1$ \\
TCho (mg/dl) & $56.3 \pm 5.8$ & $50.8 \pm 3.8$ & $230.3 \pm 22.6^{* \#}$ & $133.5 \pm 11.3^{\#}$ & $57.8 \pm 5.1^{\#}$ \\
HDL-Cho (mg/dl) & $29.6 \pm 3.0$ & $28.0 \pm 2.2$ & $78.5 \pm 13.5^{* \#}$ & $62.5 \pm 15.8$ & $29.7 \pm 4.3^{\#}$ \\
LDL-Cho (mg/dl) & $24.0 \pm 3.2$ & $21.3 \pm 1.7$ & $103.5 \pm 22.3^{* \#}$ & $53.8 \pm 11.6$ & $27.5 \pm 2.9$ \\
FFA (mEq/l) & $0.25 \pm 0.02$ & $0.14 \pm 0.02^{* \#}$ & $0.47 \pm 0.13^{\#}$ & $0.42 \pm 0.07^{*}$ & $0.40 \pm 0.08$ \\
Insulin (mg/l) & $0.022 \pm 0.010$ & $0.078 \pm 0.040$ & $0.079 \pm 0.040$ & $0.134 \pm 0.050^{*}$ & $0.021 \pm 0.010^{\#}$ \\
Cortisol (mg/dl) & $18.6 \pm 5.8$ & $11.4 \pm 4.1$ & $12.3 \pm 3.5$ & $16.3 \pm 5.7$ & $7.4 \pm 3.1$ \\
\hline
\end{tabular}

BW: Body weight, BG: blood glucose, TG: triglyceride, TCho: total cholesterol, LDL-Cho: low-density lipoprotein-cholesterol, HDL-Cho: highdensity lipoprotein-cholesterol, FFA: free fatty acids. Values are means \pm SE. Values were analyzed by Student's paired $t$-test. Significantly different at $p<0.05$ compared with *phase I, and ${ }^{\# p r e v i o u s ~ p h a s e . ~}$

of blood sampling on LA and BT values. Blood was kept for $30 \mathrm{~min}$ at room temperature and centrifuged $(1208.5 \times g$ for $15 \mathrm{~min})$, and then the supernatant was stored at $-80^{\circ} \mathrm{C}$. The measurement of blood glucose (BG), triglycerides (TG), total cholesterol (TCho), high-density lipoprotein-cholesterol (HDL-Cho), low-density lipoprotein-cholesterol (LDL-Cho), free fatty acids (FFA), and cortisol were carried out by Clinical Pathology Laboratory, Co. Ltd., Kagoshima, Japan. Insulin levels were measured using an enzymelinked immunosolvent assay kit for swine insulin (Mercodia, Uppsala, Sweden).

Data analysis. The respective treatments (phases I-V) were performed for 3 weeks. The values for LA and BT obtained from the first week were considered as the acclimation period. The values from the subsequent 2 weeks were considered for analysis. The 14 data values from the 2 weeks were averaged per pig. The BW changes during each phase (3 weeks) were evaluated (Table I). Statistical analysis was performed using paired Student's $t$-test and two-way ANOVA (repeated measurement, paired) as appropriate (Ekuseru-Toukei 2012, Social Survey Research Information; SPSS ver. 24, IBM Corp., Armonk, NY, USA). Differences with $p<0.05$ was considered significant.

\section{Results}

Effects of diet and light on BW changes. Changes in BW during the respective phases are shown in Table I. The initial and final $\mathrm{BW}$ of the four pigs used were 12.7 and $17.8,14.8$ and $22.0,16.7$ and 24.6 , and 19.5 and $27.1 \mathrm{~kg}$, respectively. The MHF diet in phase III increased the change in BW, possibly because of the higher intake of energy as part of the MHF diet. The altered BW was significantly different in phase IV under the longer duration of light, as compared with phases I and III. The longer duration of lighting may increase energy expenditures because of increased LA and BT (Figure 2).
Locomotor activity and body temperature. As shown in Figure 2A (phase I), LA and BT during the lights-on condition were significantly higher than those during the lights-off condition (LA: $8687 \pm 690 v s .4796 \pm 468$ counts; BT: $37.8 \pm 0.1 v s$. $36.9 \pm 0.1^{\circ} \mathrm{C}$ ), indicating that microminipigs are indeed diurnal animals. The increase in LA corresponded with mealtimes under the standard and MHF dietary conditions (Figure 2). The MHF diet, and longer duration of light exposure induced significantly higher LA during the nocturnal period than under standard conditions (Figure 2B and C). Under the MHF dietary conditions, microminipigs had significantly higher BT during the nocturnal period than under the standard diet, but not during the diurnal period (Figure 2B). In addition to LA, BT was also higher under longer duration of light than under standard lighting conditions; however, the difference did not reach significance (Figure 2C). Although change in BT was not as clear as compared with LA, the increase of BT corresponded to mealtimes under the standard and MHF dietary conditions (Figure 2).

When returned to the initial, standard condition (phase V, the same as phase I), LA remained higher, with a similar pattern shown in BT. The continued alteration in LA during the nocturnal period may have been a result of the obesity induced by the MHF diet, as evaluated by BW changes, but it was not associated with alterations in lipid profiles (Table I).

Blood parameters including stress hormone. Under the MHF dietary conditions (phase III compared with phase II), TCho, HDL-Cho, LDL-Cho, and FFA were significantly higher than those values under standard dietary conditions (Table I). During phase IV, the longer duration of light significantly reduced TG and TCho compared with the standard duration of lighting (phase III). Although the values did not reach 

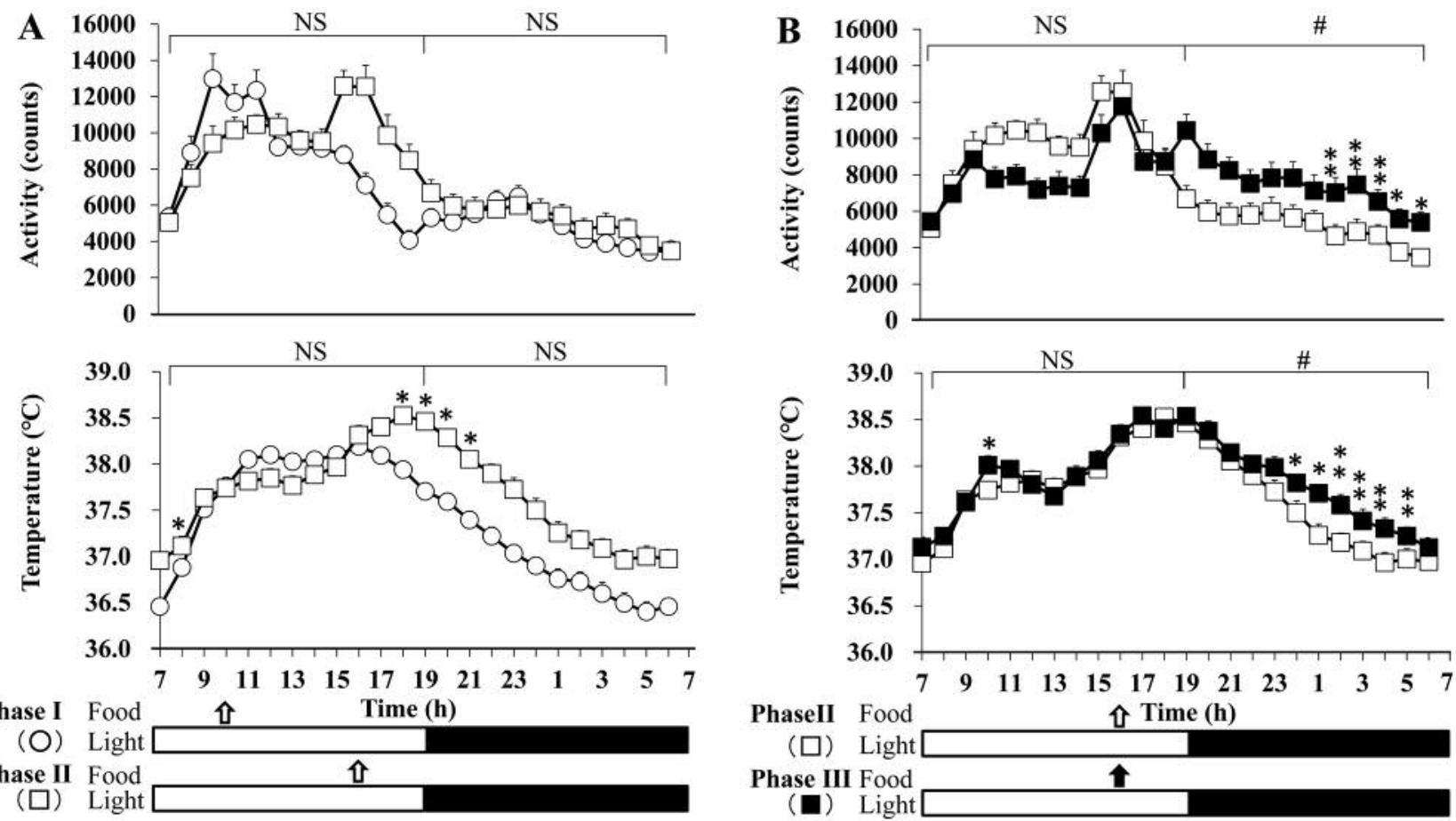

PhaseII Food

() Light

Phase III Food

(घ) Light

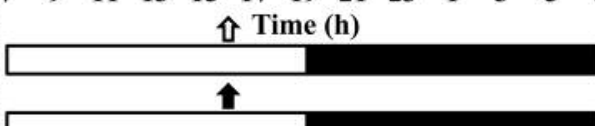

( $\square$ ) Light
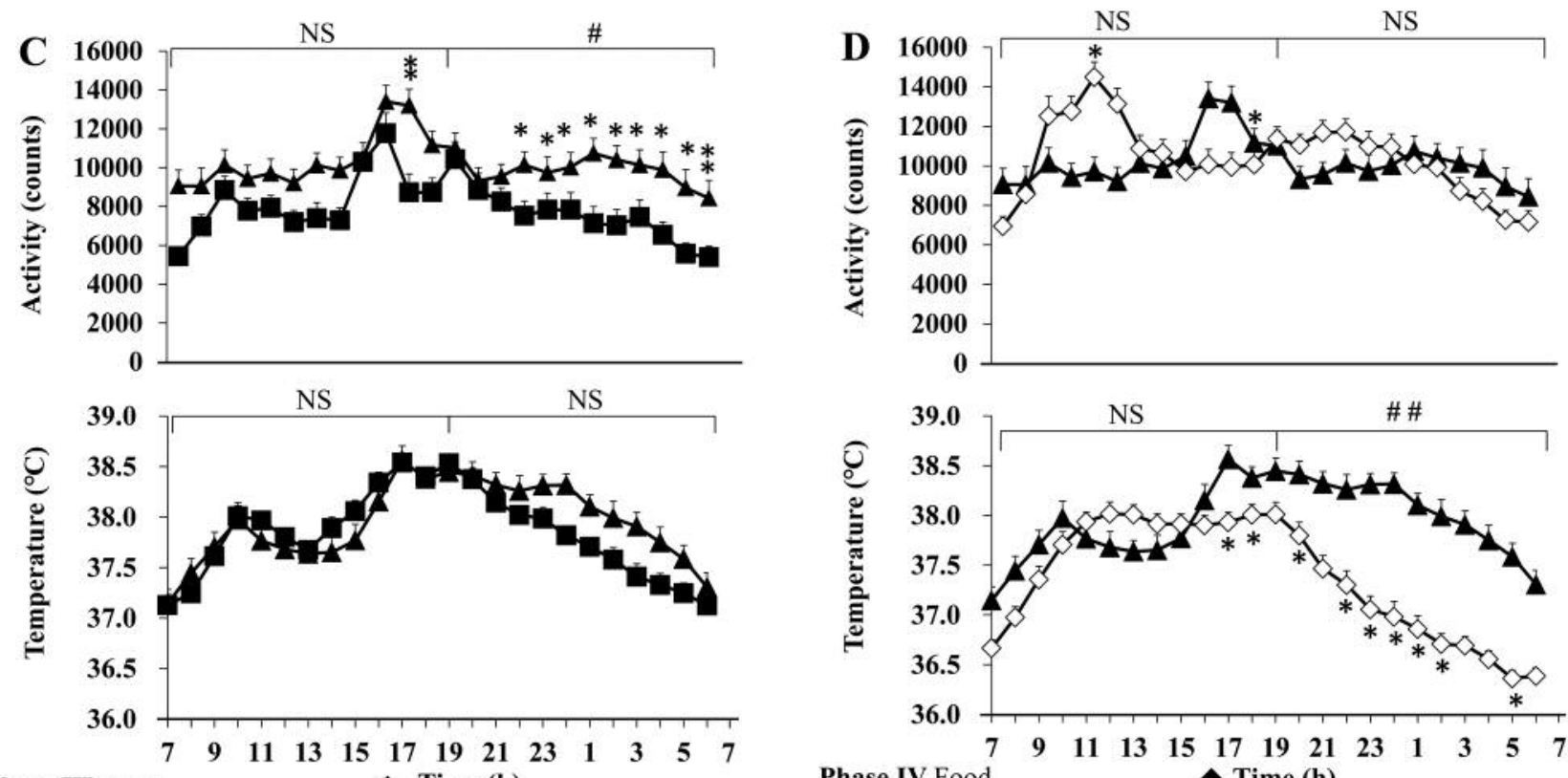

Phase III Food

1 Time (h)

Phase IV Food

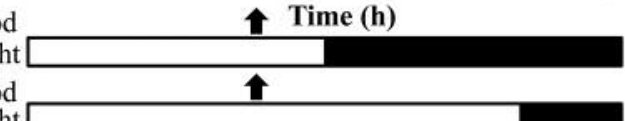

Phase IV Food

^ Time (h)

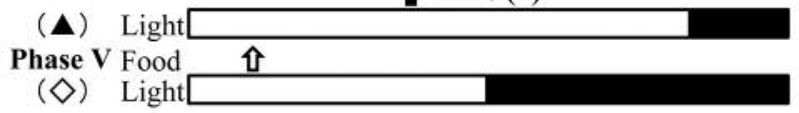

Figure 2. Continued 

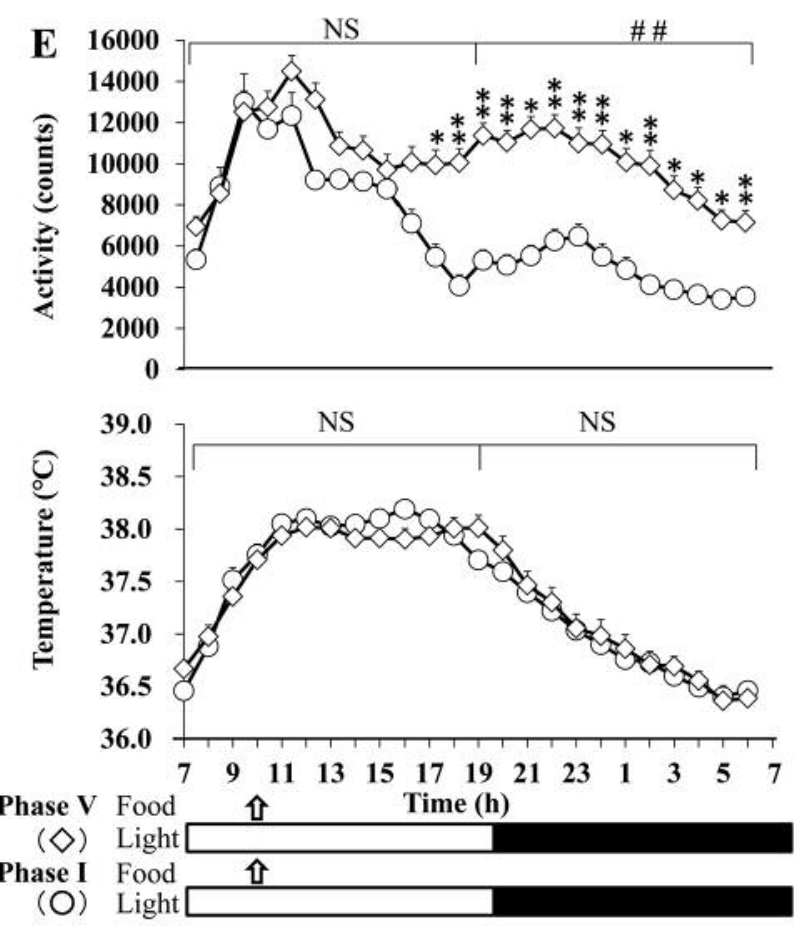

Figure 2. The effects of a moderately high-fat diet and lighting on locomotor activity (LA) and body temperature (BT) over the course of a day are shown. The values on the longitudinal axis represent the total counts per $1 \mathrm{~h}$ measured using an actigraph. The comparisons between phase I and II (A), II and III (B), III and IV $(C)$, phases IV and $V(D)$, and $I$ and $V(E)$ are shown. Dietary and lighting conditions are shown in the lower portion of the figure. Arrows indicate mealtimes (10:00 or 16:00 h). The standard and moderately high-fat dietary conditions are denoted by open and closed arrows, respectively. Light and dark conditions are represented by open and dark bars, respectively. Data values are shown as means $+S E$. Significantly different at $* p<0.05$ and ${ }^{* *} p<0.01$, between data at the same time point (Student's paired t-test), and at ${ }^{\#}<<0.05$ and ${ }^{\# \#} p<0.01$ between the different treatments with respect to lighting conditions (two-way ANOVA, repeated measurement, paired). NS: Not significantly different.

significance, BG and LDL-Cho decreased during phase IV compared with phase III. Under phase V (the same conditions as phase I), lipid metabolites including TCho, HDL-Cho, and insulin returned to values observed during phase I. In the examination of cortisol level, related to stress, there were no significant differences between the respective phases. The alterations in LA and BT during phase III, compared to phase II, were associated with elevations in blood lipid metabolites, but not with cortisol level.

\section{Discussion}

We revealed the effects of mealtime (10:00 or 16:00 h), diet ( $6 \%$ or $34 \%$ total energy from fat), and light (12- or $20-\mathrm{h}$ lights on) on LA and BT using microminipigs representing omnivorous and diurnal animals like humans. The delay of mealtime altered BT but not LA. LA and BT during the nocturnal period, but not during the diurnal period, under the MHF dietary conditions and longer light duration were significantly or relatively higher compared with those under standard conditions. The alterations in LA and BT, in part, were associated with elevation of blood lipid metabolites.

Experimental design to reduce number of animals used. The experiments were performed using four adult microminipigs. In general, female animals have additional factors related to menstruation which may be involved in changes in BT. Therefore, we used only male animals. The different conditions were applied to the same animals every 3 weeks, sequentially. Evaluating the effects of the conditions using the same animals reduced the influence of the variation of animals because the statistical analyses used paired Student's $t$-tests. Our experimental design to evaluate the effects of these factors contributes to improving animal welfare by reduction of the number of animals used.

Measurement of LA and BT. To evaluate the effects of dietary and lighting conditions on LA and BT, the methods used to measure these outcomes require high reproducibility and reliability. LA was measured using an actigraph, which was originally developed for humans (24). Recently, the use of actigraph to measure LA has been applied to other large mammals such as pigs (20). In general, LA for minipigs has been measured by a probe placed in the body using a telemetric system $(13,26)$. The LA measurements made here using an actigraph were largely consistent with the results obtained using the telemetric system. However, in the present study, the LA during the nocturnal period was higher than those previously reported $(13,26)$. This difference may be because of the different levels in sensitivity in recording LA. The higher sensitivity of actigraph in recording LA during the nocturnal period is preferred to evaluate the effects of extrinsic factors on LA. In addition, because use of an actigraph is less invasive, the procedure is safe for the animals. Other procedures for BT measurement have been reported. As a non-invasive method, tympanic membrane temperature is measured (27). However, to exclude the influence of ambient temperature, a probe placed in the body was used here. BT depends on the placement of the probe (25). In the present study, a location subcutaneously on the neck behind the ear was used for BT measurement. The present data are largely consistent with temperatures measured in the carotid artery (28), indicating that the change in BT exhibits a diurnal pattern (Figures 1 and 2). The change in BT is like that of humans $(5,6,29)$. Altogether, measurements of LA and BT were stable and consistent with data reported for large animals. 
A microminipig model for studying influences of external factors on behavior. In the present study, effects of mealtime, dietary content and duration of lighting on biological activities were examined using a novel microminipig model. The delay of mealtime and higher content of dietary fat increased BT during the nocturnal period. In addition, a higher content of dietary fat also increased LA during the nocturnal period. In modern society, delayed mealtimes, consuming high-fat diets, and spending a longer duration in lighting have been major alterations in lifestyle over the past several decades (1-4). In humans, delayed mealtimes and consuming a high-fat diet may be related to lifestyle diseases and mental health problems $(30,31)$. The present study showed the effects of lifestyle alterations on biological activity by means of microminipigs. In rodents, a high-fat diet has been reported to increase LA and BT during inactive (lights on) periods $(32,33)$. However, we should consider the dietary habits and behaviors of the respective animals when evaluating the effects of dietary and lighting conditions. Rodents continuously consume food, although mostly during the dark period (9). On the other hand, dogs are by nature carnivorous animals and may be diurnal, but the diurnal rhythm of BT is not so remarkable (13). Rodents and dogs differ from humans in dietary habits and behaviors. Swine, such as minipigs, are omnivorous and diurnal animals $(13,14,16,17)$. We suggest that minipigs are a useful model to evaluate the effects of dietary and lighting conditions on biological activities because of the similar dietary habits and behaviors to humans. The MHF diet, and a longer duration of lighting increased LA and BT during the nocturnal period (Figure 2). Increased BT during the nocturnal period may be associated with decreased sleep quality in humans (29). Some aspects regarding the influence of mealtime on sleep quality as related to stress vulnerability in humans are already known. For example, we recently reported that nurses at a university hospital consuming dinner within $2 \mathrm{~h}$ before bedtime are vulnerable to occupational stress (31). The present study was the first step in considering the significance of influences of external factors on behaviors as an animal model for humans.

Underlying mechanism of effects of dietary and lighting conditions on LA and BT. Increased BT during the nocturnal period due to delayed mealtime may account for diet-induced thermogenesis (33). The increased LA and BT during nocturnal periods under the MHF dietary conditions may have been induced by the increased energy intake (about 0.8 $\mathrm{kcal} / \mathrm{g}$ of diet) or gastrointestinal signals produced by the increased intake of the lard and cholesterol (34). Lipids can interact with intestinal cells, including epithelia and leukocytes, possibly leading to the production of cytokines involved in the regulation of LA and BT. This speculation requires further study. Additionally, the increased LA and BT were associated with decreased lipid metabolites, suggesting that increased LA and BT are associated with increased energy expenditure. This explanation is supported by the change in BW during the longer lighting period (phase IV; Figure $2 \mathrm{C}$ and Table I). Interestingly, the minipig model showed a sustained increase in LA, while BT returned to normal when pigs were returned to the initial condition, suggesting that LA differ from BT in terms of regulation. Increased LA is not always associated with increased BT in rodent models (35). The sustained increase in LA may be related to an increase in BW (Table I). Although cortisol, a stress hormone, did not significantly differe between phases $\mathrm{I}$ and $\mathrm{V}$, levels of cortisol were rather low in phase V. Because the underlying mechanism of the increase in LA is still unclear, further research is required to explore the influence of obesity.

The influence of lighting on sleepiness related to LA and BT has been reported in humans, but the alteration depends on the strength of the lighting (36). The 300 lux used in the present study may be sufficient to maintain wakefulness in pigs.

Limitations and prospective value of the study. The present study had certain limitations. Firstly, the number of animals was small and the statistical power of the analysis should be considered. However, comparison of the data between the subjects before and after treatment is effective to suppress the variation of the subject when examined in small numbers. As mentioned above, our experimental design to evaluate the effect of environmental factors contributes to improved animal welfare by reducing the number of animals used. We believe that the statistical significance obtained in the study has merit even with the small sample. We performed a power analysis using G*power software (http://www.gpower.hhu.de/) to evaluate the meaning of the statistics. Under the conditions of $\alpha=0.05, \beta=0.2, N=4$, and a paired Student's $t$-test, the effect size was calculated as 2.12. However, the present study demonstrates convincing statistics over the 2.12 effect size. Secondly, the present study was not performed using a crossover design. The experimental conditions were continuously changed, and the initial design was performed as phase V. Therefore, the statistical analysis was basically performed between two successive groups in time. The results should be interpreted cautiously given confounding factors such as environment and factors related to growth. Thirdly, food intake was not under ad libitum conditions. Our recorded changes in LA may be related to food-seeking behaviors, which are influenced by the availability of food and related to hunger (37). Therefore, we should carefully interpret the results from the restricted food conditions. In the future, comparisons between free and restricted feeding conditions should be examined. Fourthly, it is not obvious whether the duration of the observation period was sufficient 
for the evaluation of conditions. Because there is a possibility that short-term effects differ from long-term effects, a longer duration may be required to evaluate long-term effects. Fifthly, we used lard and cholesterol to prepare the MHF diet. Different kinds of fat may lead to different results. In future research, different kinds of fat, such as vegetable oil, should be examined. Lastly, the results obtained in the present study can be compared with other diurnal animals such as humans and $\operatorname{dog} s$, and are easily extrapolated to these species. However, the results are not easily extrapolated to rodents.

In conclusion, to the best of our knowledge, this is the first report evaluating the effects of dietary and lighting conditions on LA and BT as physiological outcomes in a microminipig model of omnivorous diurnal animals similar to humans. Delay of mealtime, a high-fat diet, and longer duration of lighting affected LA and BT. The present study provides an example of the effects of diet and lighting on biological activities in microminipigs under low-invasive procedures measuring LA and BT, leading to low variations in these measures.

\section{Conflicts of Interest}

There are no conflicts of interest in regard to this study.

\section{Acknowledgements}

The present research was supported by research funding from Kagoshima University. The Authors thank all the staff members of the Division of Laboratory Animal Sciences, Natural Science Center for Research and Education, Kagoshima University who kept the animals in good condition. This work was partly supported by JSPS KAKENHI Grant Numbers 25450426 (HK) and 16K08023 (HK).

\section{References}

1 Shah M, French SA, Jeffery RW, McGovern PG, Forster JL and Lando HA: Correlates of high fat/calorie food intake in a worksite population: the healthy work project. Addict Behav 18 : 583-594, 1993.

2 Szosland D: Shift work and metabolic syndrome, diabetes mellitus and ischaemic heart disease. Int J Occup Med Environ Health 23: 287-291, 2010.

3 Wirth M, Vena JE, Smith EK, Bauer SE, Violanti J and Burch J: The epidemiology of cancer among police officers. Am J Ind Med 56: 439-453, 2013.

4 Cho Y, Ryu SH, Lee BR, Kim KH, Lee E and Choi J: Effects of artificial light at night on human health: a literature review of observational and experimental studies applied to exposure assessment. Chronobiol Int 32: 1294-1310, 2015.

5 Aschoff J: Circadian control of body temperature. J Therm Biol 8: 143-147, 1983

6 Baehr EK, Revelle W and Eastman CI: Individual differences in the phase and amplitude of the human circadian temperature rhythm: with an emphasis on morningness-eveningness. J Sleep Res 9: 117-127, 2000.
7 Poyurovsky M, Nave R, Epstein R, Tzischinsky O, Schneidman M, Barnes TR, Weizman A and Lavie P: Actigraphic monitoring (actigraphy) of circadian locomotor activity in schizophrenic patients with acute neuroleptic-induced akathisia. Eur Neuropsychopharmacol 10: 171-176, 2000.

8 Kim J, Nakamura T, Kikuchi H, Sasaki T and Yamamoto Y: Covariation of depressive mood and locomotor dynamics evaluated by ecological momentary assessment in healthy humans. PLOS ONE 8: e74979, 2013.

9 Kohsaka A, Laposky AD, Ramsey KM, Estrada C, Joshu C, Kobayashi Y, Turek FW and Bass J: High-fat diet disrupts behavioral and molecular circadian rhythms in mice. Cell Metab 6: 414-421, 2007.

10 Oosterman JE, Kalsbeek A, la Fleur SE and Belsham DD: Impact of nutrients on circadian rhythmicity. Am J Physiol Regul Integr Comp Physiol 308: R337-R350, 2015.

11 Hubbard J, Ruppert E, Calvel L, Robin-Choteau L, Gropp CM, Allemann C, Reibel S, Sage-Ciocca D and Bourgin P: Arvicanthis anasorgei, a novel model for the study of sleep and waking in diurnal rodents. Sleep 38: 979-988, 2015.

12 Opperhuizen AL, van Kerkhof LWM, Proper KI, Rodenburg W and Kalsbeek A: Rodent models to study the metabolic effects of shiftwork in humans. Front Pharmacol 6: 50, 2015.

13 Yuan H, Zhao J, Guo J, Wu R, He L, Cui Y, Feng M, Zhang T, Hou M, Guo Q, Zhang L, Jia L, Huang C, Ye L and Peng S: Comparison of freely-moving telemetry Chinese miniature experiment pigs (CMEPs) to beagle dogs in cardiovascular safety pharmacology studies. J Pharmacol Toxicol Methods 70: 19-28, 2014.

14 Houpt KA, Houpt TR and Pond WG: The pig as a model for the study of obesity and of control of food intake: a review. Yale J Biol Med 52: 307-329, 1979.

15 Axelsson E, Ratnakumar A, Arendt ML, Maqbool K, Webster MT, Perloski M, Liberg O, Arnemo JM, Hedhammar A and Lindblad-Toh K: The genomic signature of dog domestication reveals adaptation to a starch-rich diet. Nature 495: 360-364, 2013.

16 Heinritz SN, Mosenthin R and Weiss E: Use of pigs as a potential model for research into dietary modulation of the human gut microbiota. Nutr Res Rev 26: 191-209, 2013.

17 Ingram DL and Mount LE: The effects of food intake and fasting on 24-hourly variations in body temperature in the young pig. Pflügers Arch 339: 299-304, 1973.

18 Miyoshi N, Horiuchi M, Inokuchi Y, Miyamoto Y, Miura N, Tokunaga S, Fujiki M, Izumi Y, Miyajima H, Nagata R, Misumi $\mathrm{K}$, Takeuchi T, Tanimoto A, Yasuda N, Yoshida $\mathrm{H}$ and Kawaguchi H: Novel microminipig model of atherosclerosis by high fat and high cholesterol diet, established in Japan. In Vivo 24: 671-680, 2010.

19 Kawaguchi H, Yamada T, Miura N, Ayaori M, Uto-Kondo H, Ikegawa M, Noguchi M, Wang KY, Izumi $\mathrm{H}$ and Tanimoto A: Rapid development of atherosclerosis in the world's smallest microminipig fed a high-fat/high-cholesterol diet. J Atheroscl Thromb 21: 186-203, 2014.

20 Takeishi K, Horiuchi M, Kawaguchi H, Deguchi Y, Izumi H, Arimura E, Kuchiiwa S, Tanimoto A and Takeuchi T: Acupuncture improves sleep conditions of minipigs representing diurnal animals through an anatomically similar point to the acupoint (GV20) effective for humans. Evid Based Complement Alternat Med 2012: 472982, 2012. 
21 Kawaguchi H, Yamada T, Miura N, Takahashi Y, Yoshikawa T, Izumi $\mathrm{H}$, Kawarasaki T, Miyoshi $\mathrm{N}$ and Tanimoto A: References values of hematological and biochemical parameters for the world smallest microminipigs. J Vet Med Sci 74: 933-936, 2012.

22 Kangawa A, Otake M, Enya S, Yoshida T, Kangawa Y and Shibata M: Spermatogenesis in the microminipig. Toxicol Pathol 44: 974-986, 2016.

23 Novotony JA, Gebauer SK and Baer DJ: Discrepancy between the Atwater factor predicted and empirically measured energy values of almonds in human diets. Am J Clin Nutr 96: 296-301, 2012.

24 Cole RJ, Kripke DF, Gruen W, Mullaney DJ and Gillin JC: Automatic sleep/wake identification from wrist activity. Sleep 15: 461-469, 1992.

25 Ikegami K, Atsumi Y, Yorinaga E, Ono H, Murayama I, Nakane Y, Ota W, Arai N, Tega A, Iigo M, Darras VM, Tsutsui K, Hayashi Y, Yoshida S and Yoshimura T: Low temperatureinduced circulating triiodothyronine accelerates seasonal testicular regression. Endocrinology 156: 647-659, 2015.

26 Myrie SB, McKnight LL, King JC, McGuire JJ, Van Vliet BN and Bertolo RF: Effects of a diet high in salt, fat, and sugar on telemetric blood pressure measurements in conscious, unrestrained adult Yucatan miniature swine (Sus scrofa). Comp Med 62: 282-290, 2012.

27 Hanneman SK, McKay K, Costas G and Rosenstrauch D: Circadian temperature rhythm of laboratory swine. Comp Med 55: 249-255, 2005

28 Ingram DL and Legge KF: Variations in deep body temperature in the young unrestrained pig over the 24 hour period. J Physiol 210: 989-998, 1970.

29 Lack LC and Lushington K: The rhythms of human sleep propensity and core body temperature. J Sleep Res 5: 1-11, 1996.
30 Astrup A: Healthy lifestyles in Europe: prevention of obesity and type II diabetes by diet and physical activity. Pub Health Nutr 4: 499-515, 2001.

31 Okita S, Daitoku S, Abe M, Arimura E, Setoyama H, Koriyama C, Ushikai M, Kawaguchi $\mathrm{H}$ and Horiuch $\mathrm{M}$ : Potential predictors of susceptibility to occupational stress in Japanese novice nurses - a pilot study. Environ Health Prev Med 22: 20, 2017.

32 Mendoza J, Pévet P and Challet E: High-fat feeding alters the clock synchronization to light. J Physiol 586: 5901-5910, 2008.

33 Yoon JA, Han DH, Noh JY, Kim MH, Son GH, Kim K, Kim CJ, Pak YK and Cho S: Meal time shift disturbs circadian rhythmicity along with metabolic and behavioral alterations in mice. PLOS ONE 7: e44053, 2012.

34 Duca FA, Sakar Y and Covasa M: The modulatory role of high fat feeding on gastrointestinal signals in obesity. J Nutr Biochem 24: 1663-1677, 2013.

35 Miyazaki K, Itoh N, Ohyama S, Kadota K and Oishi K: Continuous exposure to a novel stressor based on water aversion induces abnormal circadian locomotor rhythms and sleep-wake cycles in mice. PLOS ONE 8: e55452, 2013.

36 Cajochen C: Alerting effects of light. Sleep Med Rev 11: 453464, 2007.

37 Colpoys JD, Johnson AK and Gabler NK: Daily feeding regimen impacts pig growth and behavior. Physiol Behav 159: 27-32, 2016. 